\title{
A Study on Effectiveness of Platelet-poor Plasma as a Media in Autologous Noncultured Epidermal Cell Suspension in the Treatment of Stable Vitiligo
}

\author{
${ }^{1}$ Maraluru D Abhilasha, ${ }^{2}$ Umashankar Nagaraju
}

\begin{abstract}
Introduction: Replenishing melanocytes selectively in vitiliginous macules by autologous noncultured epidermal cell suspension (NECS) is a promising approach. Limitation of this surgery is its expensive reagents. Platelet-poor (PPP) plasma may replace these expensive reagents.
\end{abstract}

Objective: To evaluate the surgical results when two reagents, namely trypsin inhibitor and Dulbecco's modified Eagle's medium (DMEM), are replaced by patient's PPP as media in NECS.

Materials and methods: Descriptive and inferential statistical analysis has been carried out in a clinical study that enrolled 60 stable vitiligo lesions from 60 patients who formed the study group. Patients received melanocytes suspended in their own PPP.

Results: Results show that $96.7 \%$ repigmentation was achieved in 29 of 30 lesions. Additionally, 3.3\% lesions achieved poor repigmentation. Statistical analysis revealed no significant differences with respect to the repigmentation achieved or adverse effects observed.

Discussion: Trypsin inhibitor can be replaced by blood plasma as plasma contains alpha 1 antitrypsin, which covalently binds and inactivates action of trypsin. Effective results with blood plasma was possibly due to growth factors in serum which may enhance activity of melanocytes. It also enhances the viscosity of suspension.

Conclusion: Platelet-poor plasma can be one of the novel surgical modalities. It is cost-effective and beneficial for our vitiligo patients.

Keywords: Dulbecco's modified eagle's medium, Noncultured epidermal cell suspension, Platelet-poor plasma.

How to cite this article: Abhilasha MD, Nagaraju U. A Study on Effectiveness of Platelet-poor Plasma as a Media in Autologous Noncultured Epidermal Cell Suspension in the Treatment of Stable Vitiligo. J Med Sci 2018;4(2):42-47.

Source of support: Nil

Conflict of interest: None

\footnotetext{
${ }^{1}$ Junior Resident, ${ }^{2}$ Professor

1,2Department of Dermatology, RajaRajeswari Medical College \& Hospital, Bengaluru, Karnataka, India

Corresponding Author: Maraluru D Abhilasha, Junior Resident Department of Dermatology, RajaRajeswari Medical College \& Hospital, Bengaluru, Karnataka, India, e-mail: drabhi16mbbs2009 @gmail.com
}

\section{INTRODUCTION}

Vitiligo is one of the common depigmentary disorders that remains one of the major dermatological challenges worldwide, especially in India. ${ }^{1}$ It affects approximately 3 to $4 \%$ in India ${ }^{2}$ and $1 \%$ of the world's population and affects all races. ${ }^{3}$ In view of multiple hypotheses behind the pathogenesis of vitiligo, proper treatment modality has not been established. The response to medical line of treatment is $30 \%$. Surgical management may be the ray of hope in remaining patients if the vitiligo is stable. Stable vitiligo amenable to surgical modality of treatment depends on the site, age, and stability of the disease. ${ }^{3}$ For the past one decade, NECS has gained popularity because of superior esthetic results, requirement of only smaller donor area, and suitable to treat difficult areas. During the last 2 to 3 years, many modifications have been attempted to simplify the procedure and to get better outcome. ${ }^{4}$ Conventional reagents used include trypsin ethylenediaminetetraacetic acid (EDTA), trypsin inhibitor, DMEM. However, limitation of NECS technique is the high cost of these reagents.

Animal sera, especially fetal bovine serum, are an important constituent of most melanocyte culture media and are believed to be rich in melanocyte growth factors. ${ }^{5}$ Hence, use of human serum instead of conventional reagents as a suspension medium for noncultured melanocytes would give equal or better results. Recent literature has shown usage of patient's own plasma as an alternative to DMEM. ${ }^{5,6}$ The PPP also has trypsin inhibitor property. ${ }^{7}$

With this background, in this study, we attempted to replace two expensive reagents namely trypsin inhibitor and DMEM with patient's own PPP in NECS surgery in the treatment of stable vitiligo, which may be costeffective and beneficial for our vitiligo patients.

\section{MATERIALS AND METHODS}

Sample size included 30 stable vitiligo patients.

Sample size was calculated by the formula

$$
\begin{aligned}
& n=4 p q / D^{2} \\
& n=24 \text { where } p=1 \% \text { (prevalence of vitiligo) }^{6} \\
& q=(1-p)=0.09 \\
& D=\text { beta error } 15 \%
\end{aligned}
$$


Hence, the calculated sample size is 24 and for convenient purpose, we considered the sample size as 30 .

Approval of this study was obtained from the RajaRajeswari Medical College \& Hospital institutional ethics committee. Written informed consent was obtained from each participant. Patients were recruited from our dermatology clinic. They underwent NCES between December 2015 and November 2016; all patients were followed up for a maximum of 6 months. The study design was a randomized controlled clinical study. Patients older than 18 years with vitiligo and stable disease were enrolled. Disease status was based on self-reported history of no new lesions, no progression of existing lesions and absence of Koebner phenomenon during the past 1 year. Patients who reported with history of keloids, pregnant or lactating women, bleeding disorders, anemia, hematological disorders, platelet count less than 1.5 lakhs/cumm, patients on systemic steroids, anticoagulant medications, uncontrolled diabetes, and patients with unrealistic expectations were excluded.

During clinical evaluation, each patient with vitiligo was classified as having generalized, focal, or segmental involvement.

\section{PROCEDURE}

A total number of 60 stable vitiligo lesions from 30 patients were chosen.

\section{PREPARATION OF BLOOD PLASMA (GROUP I)}

Under aseptic precautions $17 \mathrm{~mL}$ of venous blood was collected in two anticoagulant acid citrate dextrose solution A (ACD) tubes (each tube with $8.5 \mathrm{~mL}$ of venous blood and $1.5 \mathrm{~mL}$ of ACD). This blood sample was centrifuged at room temperature for 15 minutes at $3000 \mathrm{rpm}$. Following centrifugation, upper two-thirds containing PPP was separated and stored in a sterile test tube.

\section{GRAFT HARVESTING}

Under strict aseptic precautions, the anterolateral aspect of the thigh was selected as the donor area. Care was taken to ensure that the donor area had no vitiligo patches. The size of the donor skin was taken as one-tenth of the recipient area. ${ }^{4}$ Under topical anesthesia (eutectic mixture of local anesthetics lidocaine, prilocaine 5\%) which was applied for 45 minutes before the surgery, ultrasplit-thickness skin graft was harvested from the donor area using a graft harvesting dermatome and sterile shaving blade. The donor area was primarily dressed with a paraffin dressing tulle followed by secondary dressing with sterile gauze.

\section{CELL SEPARATION TECHNIQUE}

The ultrasplit thickness skin graft harvested was transferred to a Petri dish containing $10 \mathrm{~mL}$ of the $0.2 \% \mathrm{w} / \mathrm{v}$ trypsin EDTA solution, epidermal side facing upward, and was incubated for 50 minutes at $37^{\circ} \mathrm{C}$.

The graft was transferred to a Petri dish containing $8 \mathrm{~mL}$ of extracted PPP with dermal side facing upward. Epidermis was separated from the dermis and the melanocyte and keratinocyte cells separation was carried out in same PPP media. Final suspension was made in a sterile test tube after centrifugation for 6 minutes. The supernatant was discarded and the pellet was further suspended in a test tube with $2 \mathrm{~mL}$ fresh PPP.

\section{TRANSPLANTATION TECHNIQUE}

The recipient site was abraded with a motor dermabrader. The endpoint of abrasion was pinpoint bleeding. Hemostasis was achieved by gentle pressure. The cell suspension was spread evenly on the dermabraded area and covered with dry collagen dressing to hold the cells applied. This was covered by secondary dressing with sterile paraffin gauze followed by sterile gauze. The area was immobilized by bandaging with Dynaplast (3M India Ltd., Bangalore, India), and the patient was advised to restrict movement at the site as much as possible. Patients were instructed to lie still in the same position for at least 1 hour to ensure cell fixation and then shifted.

Patient was started on oral antibiotics and antiinflammatory agents which were continued for 1 week. Dressing was removed on the 8 th day following surgery. All patients were subjected to narrow-band ultraviolet-B phototherapy from 2 nd week after surgery and followup was done once in 15 days for 3 months and once in a month till 6 months. Pre- and postprocedure photographs were taken in all patients at every follow-up visit.

At every follow-up visit, degree of repigmentation, color match, and adverse effect, if any, were noted. Color match was assessed by comparing with surrounding normal skin color. The efficacy of treatment was assessed by an independent investigator. Repigmentation ${ }^{4}$ was assessed as excellent (>90\%), good (70-89\%), fair $(30-69 \%)$, and poor $(<29 \%)$. Color match $^{4}$ was also rated as excellent (minimal hypo- or hyperpigmentation of grafted area not requiring cosmetic camouflage), good (moderate hypo- or hyperpigmentation amenable to light camouflage), and poor (gross hypo- or hyperpigmentation difficult to cover up with ordinary make-up).

Descriptive and inferential statistical analysis has been carried out in the present study. Results on continuous measurements are presented on mean e SD (min-max) and results on categorical measurements are presented in number (\%). Significance is assessed at $5 \%$ 
level of significance. Student $t$ test and chi-square/Fisher's exact test has been used to find the significance of study parameters on categorical scale between two groups. The Statistical software, namely Statistical Package for the Social Sciences version 18.0 and $\mathrm{R}$ environment version 3.2.2, were used for the analysis of the data and Microsoft word and Excel were used to generate graphs and tables.

\section{RESULTS}

The maximum number of patients, 13 (43.3\%), were found to be in the age group 21-25 years. In the study, males, $20(66.7 \%)$, outnumbered females, 10 (33.3\%), with maleto-female ratio of $2: 1 ; 26.7 \%$ had segmental vitiligo, $23.3 \%$ had mixed vitiligo, $20 \%$ had vitiligo vulgaris, $16.7 \%$ had focal vitiligo, and $6.7 \%$ had mucosal and acrofacial vitiligo each (Table 1). Onset of repigmentation was seen as early as 2 weeks in $33.3 \%$ of lesions postoperatively and remaining $63.3 \%$ of lesions were found to start to repigment between 2 and 4 weeks in both the groups (Fig. 1). Our study showed superior results with excellent repigmentation being seen in $96.7 \%$ of lesions and poor repigmentation in $3 \%$ of lesions at the end of 3 months in

Table 1: Characteristics and disease parameters of the patients in the two groups

\begin{tabular}{|c|c|c|}
\hline Characteristic & (Plasma) & $p$-value \\
\hline Patients, $\mathrm{n}$ & 30 & \\
\hline Lesions, $\mathrm{n}$ & 30 & \\
\hline Age, mean \pm SD (range) & $24.87 \pm 5.48$ & $p=1.000$ \\
\hline Male: female & $2: 1$ & $p=1.000$ \\
\hline $\begin{array}{l}\text { Duration of disease, years, } \\
\text { mean } \pm \text { SD }\end{array}$ & $6.23 \pm 3.19$ & $p=1.000$ \\
\hline $\begin{array}{l}\text { Duration of stability, years, } \\
\text { mean } \pm S D\end{array}$ & $3.82 \pm 2.51$ & $p=1.000$ \\
\hline $\begin{array}{l}\text { Type of vitiligo (generalized/focal/ } \\
\text { segmental) }\end{array}$ & $17 / 5 / 8$ & $p=1.000$ \\
\hline
\end{tabular}

Table 2: Comparison of results based on degree of repigmentation at the end of 3 months

\begin{tabular}{ll}
\hline Repigmentation & Plasma \\
\hline Excellent $(\geq 90 \%)$ & $29(96.7 \%)$ \\
Good $(70-89 \%)$ & $0(0 \%)$ \\
Fair $(30-69 \%)$ & $0(0 \%)$ \\
Poor $(<30 \%)$ & $1(3.3 \%)$ \\
Total number of vitiligo macules & $30(100 \%)$ \\
\hline$p=1.000$, not significant, Fisher's exact test &
\end{tabular}

Table 3: Comparison of results based on color matching at the end of 3 months

\begin{tabular}{ll}
\hline Color match & Plasma \\
\hline Excellent & $27(90 \%)$ \\
Good & $2(6.7 \%)$ \\
Poor & $1(3.3 \%)$ \\
Total & $30(100 \%)$ \\
\hline
\end{tabular}

$p=1.000$, not significant, Fisher's exact test

both the groups (Table 2). In the present study, 27 (90\%) showed excellent, 2 (7\%) showed good, and $1(3 \%)$ showed poor color match (Table 3 ) as that of the surrounding normally pigmented skin. The results with respect to onset of pigmentation, degree of repigmentation, and color match in both the groups using PPP and conventional reagents were statistically similar. We observed perigraft halo, hyperpigmentation, and hypopigmentation in one patient each in both the groups in our study as adverse effects.

\section{DISCUSSION}

Vitiligo is an acquired chronic pigmentation disorder characterized by white patches, often symmetrical, corresponding to a substantial loss of functioning epidermal and sometimes follicular melanocytes. Medical treatments are ineffective in many patients and surgical management has evolved significantly giving a ray of hope in stable vitiligo. Stability is taken as the most important
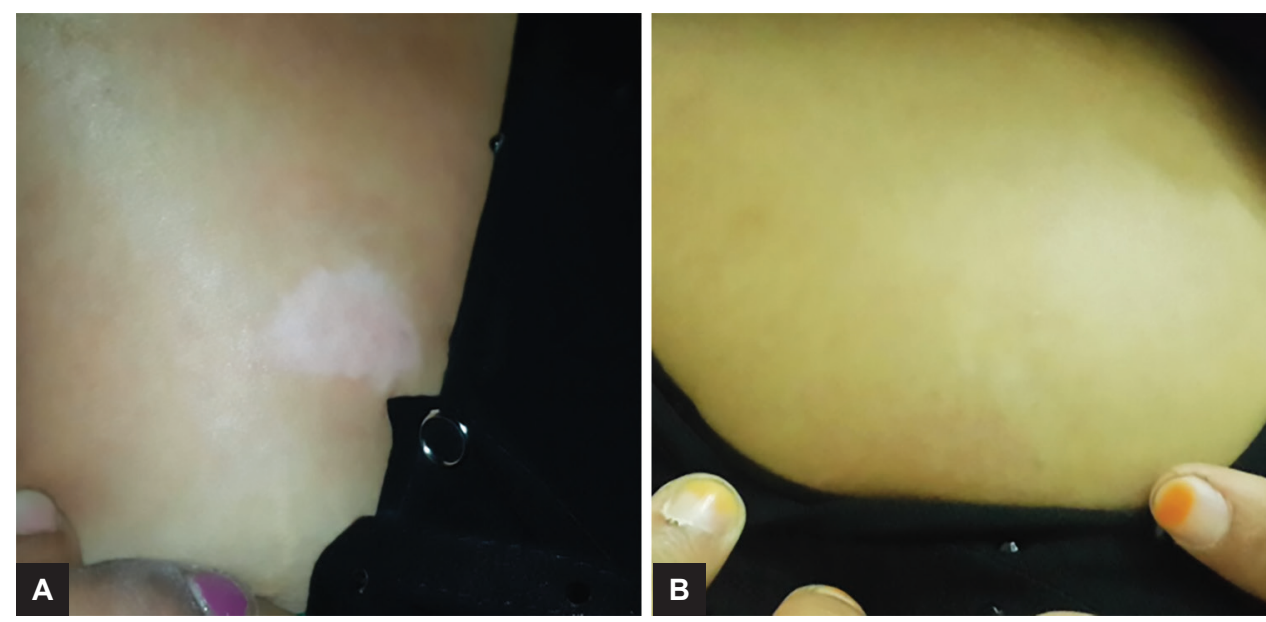

Figs 1A and B: (A) Preoperative photograph of a lesion of stable vitiligo over upper chest treated with noncultured melanocyte suspension prepared in plasma. (B) Postoperative photograph of the same lesion 12 weeks after surgery 
parameter before opting for any transplantation technique to treat vitiligo. Various tissue-grafting techniques like split skin grafting, miniature punch grafting, and blister grafting have been well documented. However, they all have limitations, such as cobble-stoning, perilesional halo, or limitations in regard to area.

Meta-analysis of various studies of surgical treatment for vitiligo revealed an average success rate of $61.1 \%$ (95\% confidence interval $=56.1-66.1 \%$ ) using noncultured melanocyte transplantation. ${ }^{8}$ They are effective modalities wherein large recipient areas can be grafted with relatively minimal donor area.

The NCES method for the management of stable vitiligo was pioneered by Gauthier and Surleve-Bazeille 9 in 1992.

The study groups in the present research did not differ with respect to demographic and disease characteristics. Lesions in both the groups were located in classically responsive and difficult-to-treat areas, and there was no statistically significant difference between the two groups.

In a study by Munish, ${ }^{4}$ Van Geel et al, ${ }^{10}$ Sobhy et $\mathrm{al}^{11}$ on stable vitiligo lesions treated by NCES using DMEM and trypsin inhibitor, the time for onset of repigmentation for the treated areas ranged between 2 and 3 weeks postoperatively. In the present study, onset of repigmentation was seen as early as 2 weeks postoperatively in both the groups.

In a study by Van Geel et $\mathrm{al}^{10}$ among 19 patients of stable vitiligo, mean percentage of repigmentation achieved was 84 and $88 \%$ at the end of 3 and 6 months respectively. In a study done by Munish, ${ }^{4}$ of 49 patients treated, $32(65 \%)$ had excellent repigmentation at the end of 6 months. Good results were seen in $9(18 \%)$, fair in $4(8 \%)$, and poor repigmentation in $4(8 \%)$ patients. In a study done by Sobhy et $\mathrm{al}^{11}$ among 20 stable vitiligo patients treated, 35\% excellent, 55\% good, 10\% fair, and $0 \%$ poor repigmentation with 6-month follow-up period were reported. In a study done by Huggins et al, ${ }^{12}$ out of 29 treated lesions, excellent repigmentation was observed in $17 \%$, good repigmentation in 31\%, fair in $10 \%$, and poor in $41 \%$ with 6 -month follow-up period. Our study showed superior results to the above studies with excellent repigmentation being seen in $96.7 \%$ of lesions and poor repigmentation in 3\% of lesions at the end of 3 months in both the groups.

Munish's ${ }^{4}$ study revealed excellent color matching in $79 \%$ patients. Color match was same as that of the surrounding normally pigmented skin in majority of patients, $90.0 \%$, in a study by Sobhy et $\mathrm{al}^{11}$ conducted among 20 stable vitiligo patients. In a study done by Huggins et $\mathrm{al}^{12}$ with 29 treated lesions, good color match was noted in $62 \%$ of cases. In a study by Van Geel et $\mathrm{al}^{10}$ among 19 patients of stable vitiligo, 5 responding lesions showed hyperpigmentation and all other lesions showed good color matching. However, in the present study, 90\% showed excellent, 7\% showed good, and 3\% showed poor color match as that of the surrounding normally pigmented skin.

Gauthier and Benzekri, ${ }^{13}$ in 2012, have suggested to use plasma as a vehicle for the cell suspension. Majid, ${ }^{6}$ in 2013, in his review of literature, has suggested some modifications in ECS surgery, one of which is replacement of trypsin inhibitor and DMEM by patient's own serum.

Sahni et $\mathrm{al}^{5}$ in 2011 conducted a study to compare the repigmentation results in stable vitiligo of transplantation of autologous noncultured epidermal suspension suspended in normal saline with that of suspension in patient's own plasma. Among 25 patients with 36 lesions treated, the time of initial repigmentation for the treated areas ranged between 2 and 3 weeks postoperatively and was excellent at the end of 4 months which was comparable with the present study among both the groups. They also found $88.8 \%$ excellent repigmentation in patients receiving their plasma as a media compared with normal saline which showed excellent repigmentation in $44.4 \%$ at the end of 16 weeks.

In $83.3 \%$ of lesions in each group, the color match of the repigmented area was excellent with the normal surrounding skin. ${ }^{5}$

Results observed in our study where PPP was used were comparable with the study done by Sahni et $\mathrm{al}^{5}$ in their plasma group.

There was no significant difference based on adverse events. Adverse events observed in the present study were perigraft halo, hyperpigmentation, and hypopigmentation in one patient each in both the groups. In a study by Sobhy et al $^{11}$ among 20 stable vitiligo patients using conventional reagents in NECS, hyperpigmentation was noted in one patient, another patient developed hypopigmentation, and perigraft halo was seen among two patients. Munish ${ }^{4}$ in his study using conventional reagents has documented hyperpigmentation in 6, hypopigmentation in 4 , and perigraft halo in 12 patients out of 49 patients. In Van Geel et al's study ${ }^{10}$ among 19 patients of stable vitiligo, 5 responding lesions showed hyperpigmentation. Perigraft halo and infection at recipient site were observed in two patients each out of 12 patients in plasma group in Sahni et al's ${ }^{5}$ study. Sobhy et $\mathrm{al}^{14}$ have hypothesized perigraft halo to residual activity of some melanocyte-destroying factors. Another explanation could be an inflow of keratinocytes from the pigmented border which does not allow the transplanted pigment cells to remain where they were seeded. This influx of keratinocytes at the margins appeared as a white 
rim which could be treated by increasing dermabrasion beyond lesion boundary during the operation session or performing another session of transplantation of frozen autologous melanocytes not used in first session.$^{15}$

In our study we used patient's plasma instead of serum. Plasma is the liquid part of blood, i.e., whole blood after the removal of red blood cells, white blood cells, and platelets by centrifuging. Serum is the part of blood which is similar in composition with plasma, but excludes clotting factors of blood. Plasma also contains thousands of different proteins and other substances (electrolytes, for example) that are nutrients for melanocytes. ${ }^{16}$

Plasma contains 95\% water, 6-8\% dissolved proteins, growth factors, $1 \%$ dissolved proteins, albumin, globulin, and fibrinogen, $2 \%$ nutrients, amino acids, sugars, and lipids hormones (insulin, erythropoietin, etc.), and electrolytes (sodium, potassium, and calcium, etc.). ${ }^{16}$ The DMEM contains inorganic salts, amino acids, vitamin D glucose, and phenol red.

Presence of growth factors like transforming growth factor, vascular endothelial growth factor, epidermal growth factor, hepatocyte growth factor, and fibroblast growth factor ${ }^{17}$ in plasma may also enhance the proliferation and activity of melanocytes. Thus, we hypothesized that replacing DMEM by blood plasma as a NECS media would improve the repigmentation. Maintenance of epidermal cells in blood plasma may also be enhanced by providing suitable nutrition, media stability, and cell performance. This is also clearly demonstrated in this study by increased viability of melanocytes in plasma.

Plasma also contains clotting factors, fibrinogen, ${ }^{17}$ which may help in faster wound healing by enhancing the clotting mechanism on dermabraded bed in recipient area. Blood plasma also enhances the viscosity of cellular suspension and prevents run-off of the suspension from the dermabraded recipient site.

A recent study Szabad et al ${ }^{18}$ demonstrated that the autologous human serum of patients with vitiligo was found to be superior to the fetal bovine serum in supporting the in vitro growth of melanocytes in culture. Sahni et $\mathrm{al}^{5}$ have hypothesized that using patient's serum as a diluent during preparation of noncultured melanocyte suspension would improve the repigmentation results through a two-pronged action: first by increasing the viscosity of the suspension and second by providing melanocyte growth factors. This was clearly proved in our study.

Trypsin EDTA is a serine protease that cleaves the protein binding between the cells. Trypsin inhibitor prevents its further enzymatic action. Blood plasma contains alpha 1 antitrypsin, a serine protease inhibitor, which also covalently binds and irreversibly inactivates the enzymatic action of trypsin. ${ }^{7}$ In 1922 conducted a study on determination of trypsin inhibitor in blood plasma and found the presence of trypsin inhibitor property in plasma. ${ }^{7}$ This was clinically shown in our study where our results in plasma group without trypsin inhibitor were similar to the DMEM group with trypsin inhibitor.

The concern that autoantibodies contained in the patients' serum may hamper melanocyte survival may be put to rest by the hypothesis that the level of autoantibodies in patients with "stable" disease may be low and below a critical level. ${ }^{19}$ This was clearly demonstrated in an animal model study on vitiligo in Smyth Chicken. Austin et $\mathrm{al}^{20}$ and Searle et $\mathrm{al}^{21}$ have shown that autoantibodies specific to melanocytes in the serum of Smyth line chickens developed immediately before or at the time of feather amelanosis and when the chickens spontaneously developed complete repigmentation, these serum autoantibodies were no longer detectable.

This study also throws light on the possibility of plasma to be a better choice than serum. However, this needs to be established further by comparative studies using plasma and serum as suspension media in NECS in vitiligo.

\section{CONCLUSION}

We emphasize that blood plasma can replace trypsin inhibitor, DMEM media, and also increases the viscosity of cell suspension. Hence, plasma can be equivalent or better than conventional reagents in obtaining excellent repigmentation following epidermal cell suspension in vitiligo patients. We believe that the modifications described here will be useful to make this procedure easy, safe, economical, and effective in the management of stable vitiligo, particularly in difficult-to-treat areas.

\section{REFERENCES}

1. Anstey AV. Disorders of skin. In: Burns T, Breathnach S, CoxN, Griffiths C, editors. Rook's textbook of dermatology. 8th ed. UK: Wiley-Blackwell; 2010. pp. 46-49.

2. Shah H, Mehta A, Astik B. Clinical and sociodemographic study of vitiligo. Indian J Dermatol Venereol Leprol 2008 Nov-Dec;74(6):701.

3. Hassan I, Mubashir S, Abdullah Z, Sajad P, Anwar P, Sheikh G, Sami A, Yaseen A. Autologous non cultured epidermal cell suspension in case of resistant segmental vitiligo: a preliminary study. J Pak Acad Dermatol 2013;23(2): 190-193.

4. Munish P. Autologous non-cultured basal cell-enriched epidermal cell suspension transplantation in vitiligo: Indian experience. J Cutan Aesthet Surg 2011 Jan-Apr;4(1): 23-28.

5. Sahni K, Prasad D, Kanwar AJ, Mehta SD. Autologous noncultured melanocyte transplantation for stable vitiligo: can suspending autologous melanocytes in the patients' own 
serum improve repigmentation and patient satisfaction? Dermatol Surg. 2011 Feb;37(2):176-182.

6. Majid I. Grafting in vitiligo: how to get better results and how to avoid complications. J Cutan Aesthet Surg Apr-Jun 2013;6(2):83-89.

7. Shirley F, Canni MC, Laskowski. Determination of trypsin inhibitor in blood plasma. J Biol Chem. 1953;19:147.

8. Gupta S, Narang T, Olsson MJ, Ortonne JP. Surgical management of vitiligo and other leukodermas: evidence-based practice guidelines. In: Gupta S, Olsson MJ, Kanwar AJ, Ortonne JP, editors. Surgical management of vitiligo. 1st ed. Massachusetts: Blackwell Publishing; 2007. pp. 69-79.

9. Gauthier Y, Surleve-Bazeille JE. Autologous grafting with non cultured melanocytes: a simplified method for treatment study of depigmented lesions. J Am Acad Dermatol 1992 Feb;26(2):191-194.

10. Van Geel NV, Ongenae K, Mil MD, Naeyaert JM. Double blind placebo-controlled study of autologous transplanted epidermal cell suspension for repigmenting vitiligo. Arch Dermatol 2004 Oct;140(10):1203-1208.

11. Sobhy N, Atia A, Elramly M. Some modifications in transplantation of autologous non-cultured melanocyteskeratinocytes suspension in treatment of segmental and focal vitiligo (Egyptian experience in Alexandria university). Our Dermatol Online 2013;4(1):5-10.

12. Huggins RH, Henderson MD, Mulekar SV, Ozog DM, Kerr HA, Jabobsen G, Lim HW, Hamzavi IH. Melanocytekeratinocyte transplantation procedure in the treatment of vitiligo: the experience of an academic medical center in the United States. J Am Acad Dermatol 2012 May;66(5):785-793.

13. Gauthier Y, Benzekri L. Non-cultured epidermal suspension in vitiligo: from laboratory to clinic. Indian J Dermatol Venereol Leprol 2012 Jan-Feb;78(1):59-63.
14. Sobhy N, Elbeheiry A, ELramly M, Saber H, Kamel L, Soror O. Transplantation of non cultured melanocytes vs dermabration and split thickness grafting in cases of non progressive vitiligo and stable forms of leucoderma [MD thesis]. Alexandria Dermatology Department; 2002.

15. Olsson M, Juhlin L. long-term follow-up of leukoderma patients treated with transplants of autologous cultured melanocytes, ultrathin epidermal sheets and basal cell layer suspension. Br J Dermatol 2002;47:893-904.

16. Hall JE. Chapter 25: Body fluid compartments, extracellular and intracellular fluids. In: Hall JE, editor. Guyton and hall textbook of medical physiology. 13th ed. Philadelphia (PA): Elsevier; 2015. p. 287.

17. Arshdeep, Kumaran MS. Platelet-rich plasma in dermatology: boon or a bane? Indian J Dermatol Venereol Leprol 2014 Jan-Feb;80(1):5-14.

18. Szabad G, Kormos B, Pivarcsi A, Széll M, Kis K, Kenderessy Szabó A, Dobozy A, Kemény L, Bata-Csörgo Z. Human adult epidermal melanocytes cultured without chemical mitogens express the EGF receptor and respond to EGF. Arch Dermatol Res 2007 Jul;299(4):191-200.

19. Pandya V, Parmar KS, Shah BJ, Bilimoria FE. A study of autologous melanocyte transfer in treatment of stable vitiligo. Indian J Dermatol Venereol Leprol 2005 Nov-Dec;71(6):393-397.

20. Austin LM, Boissy RE, Jacobson BS, Smyth JR Jr. The detection of melanocyte autoantibodies in the Smyth chicken model for vitiligo. Clin Immunol Immunopathol 1992 Aug;64(2): 112-120.

21. Searle EA, Austin LM, Boissy YL, Zhao H, Nordlund JJ, Boissy RE. Smyth chicken melanocyte autoantibodies: crossspecies recognition, in vivo binding, and plasma membrane reactivity of the antiserum. Pigment Cell Res 1993 Jun;6(3): 145-157. 